Leyton, M.; Batista, M.; Lobato, S. y Jiménez, R. (2019) Validación del cuestionario del modelo transteórico del cambio de ejercicio físico / Validation of the Questionnaire of the Transtheoretical Model of Change of Physical Exercise. Revista Internacional de Medicina y Ciencias de la $\begin{array}{lllllll}\text { Actividad Física y el Deporte vol. } 19 & \text { (74) pp. 329-350 }\end{array}$ Http://cdeporte.rediris.es/revista/revista74/artvalidez1026.htm DOI: http://doi.org/10.15366/rimcafd2019.74.010

\title{
ORIGINAL
}

\section{VALIDACIÓN DEL CUESTIONARIO DEL MODELO TRANSTEÓRICO DEL CAMBIO DE EJERCICIO FÍSICO}

\section{VALIDATION OF THE QUESTIONNAIRE OF THE TRANSTHEORETICAL MODEL OF CHANGE OF PHYSICAL EXERCISE}

\author{
Leyton, M. ${ }^{1}$; Batista, M. ${ }^{2}$; Lobato, S. $^{3}$ y Jiménez, R. ${ }^{4}$ \\ 1 Profesora Ayudante Doctora. Universidad Rey Juan Carlos. Madrid (España) \\ marta.leyton@urjc.es \\ 2 Doctor en Ciencias de la Educación. Profesor adjunto en el Instituto Politécnico de Castelo \\ Branco, Universidad de Castelo Branco (Portugal) marco.batista@ipcb.pt \\ 3 Doctora en Ciencias de la Actividad Física y del Deporte. Universidad de Extremadura \\ (España) susanalobato@gmail.com \\ ${ }^{4}$ Profesora Titular en la Facultad de Ciencias del Deporte. Universidad de Extremadura, \\ (España) ruthii@unex.es
}

Código UNESCO / UNESCO code: 3212 Salud Pública / Public Health, 3206. Clasificación Consejo de Europa / Council of Europe classification: 17. Otras: Actividad Física y Salud / Other: Physical Activity and Health

Recibido 9 de julio de 2017 Received July 9, 2017

Aceptado 1 de diciembre de 2017 Accepted December 1, 2017

\section{RESUMEN}

El objetivo de este estudio fue traducir y validar al español el Cuestionario del Modelo Transteórico del Cambio de Ejercicio Físico de Prochaska y DiClemente (1983). Se utilizó una muestra de 812 personas, entre los 14 y los 88 años $(29,5 \pm 21,7)$. Se realizó un análisis factorial confirmatorio, análisis de consistencia interna y validez predictiva. Los resultados del Cuestionario del Modelo Transteórico del Cambio de Ejercicio Físico presentaron valores adecuados $\left(x^{2} / g \cdot I=4,3, \mathrm{CFI}=0,92, \mathrm{IFI}=0,92, \mathrm{TLI}=0,90, \mathrm{RMSEA}=0,06, \mathrm{SRMR}\right.$ $=0,05)$. La consistencia interna mostró valores encima de .70. Se halló una predicción positiva y significativa de los estadios más activos del Modelo Transteórico sobre la intención de ser físicamente activo. Este estudio ha permitido proporcionar un cuestionario válido y fiable para evaluar el estadio en el que se encuentran las personas con respecto a la práctica de ejercicio físico, 
en el ámbito español.

PALABRAS CLAVES: Modelo transteórico, ejercicio físico, intención de ser físicamente activo, instrumento de medida.

\section{ABSTRACT}

The aim of this study was to translate and validate in Spanish the Questionnaire of the Transtheoretical Model of Change of Physical Exercise, of Prochaska and DiClemente (1983), and also to make adaptations and modifications as needed. The sample was composed of 812 people, aged between 14 and 88 years (29.5 \pm 21.7$)$. Confirmative factorial analysis, analysis of internal consistency and of predictive validity were carried out. After the confirmative factorial analysis, the Questionnaire of the Theoretical Model of Change of Physical Exercise showed acceptable results $(\mathrm{x} 2 / \mathrm{g} .1=4,3, \mathrm{CFI}=0,92, \mathrm{IFI}=0.92, \mathrm{TLI}=0.90$, RMSEA $=0.06$, SRMR $=0,05$ ). Similarly, internal consistency obtained from the respective dimensions showed values above .70. A positive and significant prediction of the most active stages of the Transtheoretical Model (action and maintenance) was found on the 'intention to be physically active'. This study has enabled the provision of a valid and reliable questionnaire.

KEYWORDS: Transtheoretical model, physical exercise, intention to be physically active, measuring instrument.

\section{INTRODUCCIÓN}

En la actualidad es notable el interés que suscita la práctica de ejercicio físico. Según Moreno, Pardo, Jorge, y Huéscar (2016), durante las últimas tres décadas, la práctica deportiva de la población española entre 15 y 65 años ha aumentado considerablemente, pasando de un $25 \%$ en 1980 a un $45 \%$ durante el año 2010, creciendo así el número de personas mayores (55 años o más) que practican algún tipo de actividad físico-deportiva, siendo especialmente importante la subida de la última década, y, la población joven que también viene reflejando un aumento paulatino de práctica deportiva.

Por lo tanto, se hace fundamental analizar los diferentes aspectos que pueden influir en el inicio o mantenimiento de la práctica de ejercicio físico de forma regular, debido a los importantes beneficios que dicha actividad viene demostrando (Moreno, Silva, Pardo, Rodríguez, y Hernández, 2016).

El Modelo Transteórico (MT), inicialmente fue el producto central del análisis comparativo de veintinueve de las teorías relevantes y disponibles a finales de los años setenta para explicar cambios en el comportamiento de dependientes de drogas y consumidores de cigarrillo. El modelo derivó su nombre de la integración de los principios y componentes teóricos de los diferentes sistemas de intervención analizados (Prochaska, 1979).

Otros análisis posteriores de teorías y modelos del comportamiento 
humano, así como observaciones del cambio espontáneo e inducido del comportamiento de consumidores de tabaco, y validaciones empíricas de propuestas de intervención para facilitar el abandono del consumo de sustancias generadoras de dependencia, fueron analizados como evidencias sobre la naturaleza de las etapas y los procesos que subyacen al cambio comportamental (Prochaska y DiClemente, 1982). Estos análisis preliminares de la aplicación en la práctica del MT (especialmente con relación al consumo de tabaco y sobre cómo las personas cambiaban su comportamiento de fumar por voluntad propia o como consecuencia de intervenciones externas planeadas terapéuticamente) fueron los primeros esfuerzos por aislar y describir con mayor precisión los componentes y etapas básicas del aún parcialmente desconocido proceso de cambio de este comportamiento de riesgo para la salud (Prochaska y DiClemente, 1984).

A mediados de los años ochenta, nuevos avances en la teoría y práctica generados por diversos grupos de investigación con intereses en la aplicación de modelos explicativos del comportamiento del consumo de tabaco y de los comportamientos relacionados con la salud, llevaron a la incorporación en el MT de los constructos de autoeficacia, tentación y balance decisorio. Esto ocurrió para reforzar la capacidad de las etapas y procesos de cambio para evaluar y predecir los cambios espontáneos e inducidos en el comportamiento de consumo de cigarrillo (Prochaska y Velicer, 1997).

En los primeros años de los noventa, en Estados Unidos de América y algunos países de Europa, el MT fue progresivamente incorporado en las investigaciones e intervenciones de un amplio número de comportamientos ya reconocidos de riesgo para la salud (Prochaska et al., 1994). Diversas publicaciones reconocían la capacidad del modelo para describir y explicar las distintas etapas que son comunes a la mayoría de procesos de cambio comportamental (Prochaska y Velicer, 1997). A este respecto, se destaca la revisión realizada por Guiraos, Cabrero, Moreno y Muñoz (2009) que avala la fiabilidad y validez del instrumento de medida de Marcus, Selby, Niaura, y Rossi (1992), así como los estudios de Fahrenwald y Walker (2003), Cheung, et al. (2006), entre otros, que mostraron su utilidad de aplicación en programas de intervención de práctica de ejercicio físico.

Actualmente el Modelo Transteórico del Cambio del Ejercicio Físico (MTCEJ) es usado en el diseño de intervenciones facilitadoras de comportamientos saludables como la adopción de práctica de ejercicio físico (Esparza, et al., 2016). El MTCEJ ha sido empleado en varias investigaciones de cambio de comportamiento dentro de las cuales, se pueden resaltar las de González et al., (2015a), Guzmán, Ricardo, Retamozo y Soto (2015), Práxedes, Sevil, Moreno, del Villar y García (2016), y Puigarnau, Foguet, Balcells, Ambrós y Anguera (2016), para el ejercicio físico.

El MT, supone, actualmente, uno de los modelos más prometedores en cuanto a la compresión y promoción del cambio de conducta relacionado con la adquisición de hábitos de vida saludables (DiClemente y Prochaska, 1982; Prochaska y DiClemente, 1983; Prochaska, Norcross, Fowler, Follnick y Abrams, 1992). La idea central del MT se halla en el constructo de estadio de 
cambio, que define una dimensión temporal de seis estadios (Gustavo y Cabrera, 2000; Prochaska y DiClemente, 1983): precontemplación (etapa en la cual las personas no tienen intención de cambiar), contemplación (etapa en la que la persona tiene una intención de cambio y, potencialmente, hará un intento formal de modificar su comportamiento en los próximos meses), preparación (etapa en la que las personas ya toman una decisión de cambio y tienen un compromiso propio para hacerlo; realizan pequeños cambios e intentan efectivamente cambiar en el futuro inmediato, regularmente los próximos 30 días), acción (etapa en la que las personas realizan cambios objetivos, mesurables y exteriorizados de su comportamiento, regularmente en un período de tiempo que varía de uno a seis meses) y mantenimiento (se caracteriza por las tentativas de estabilización del cambio comportamental ya manifestado).

El concepto de etapa se ha reevaluado debido al criterio de temporalidad de las etapas y a las etiquetas de cada una de ellas, sin embargo, el concepto de niveles o grados de disposición al cambio que varían de acuerdo con el grado de motivación para abandonar o cambiar una conducta ha recibido amplia evidencia empírica (Davidson, 2001; DiClemente, 2005). Los estudios han pretendido establecer con alguna precisión cuáles son los procesos propuestos por el MT que deben privilegiarse para favorecer el cambio, en función de la etapa en la que se ubique a los sujetos, especialmente para la conducta de fumar (DiClemente, et al., 1991; Herzog, Abrams, Emmons, Linnan y Shadel, 1999; Perz, DiClemente y Carbonari, 1996; Segan, Borland y Greenwood, 2004), y para otras conductas (Armitage, Sheeran, Conner y Arden, 2004). Rosen (2000) sometió a revisión 47 investigaciones en las que se plantea el uso diferencial de procesos de cambio en función de la etapa motivacional, para el comportamiento de fumar y para otros comportamientos; como conclusión propone que si bien es válida la diferenciación entre los procesos a través de las diferentes etapas, la secuenciación entre los procesos no es igual a través de diferentes conductas: en el caso de la modificación del tabaquismo y del consumo de otras sustancias se ha determinado que primero se modifican los procesos cognitivo-afectivos (procesos experienciales) para culminar en los procesos conductuales, los cuales se observan más en las etapas de acción y de mantenimiento. En cambio, para otros comportamientos como el ejercicio físico y la dieta, el uso de procesos cognoscitivos y conductuales ha sido más simultáneo a lo largo de todas las etapas (FlórezAlarcón, 2005). Parcialmente se ha atribuido esa diferencia en el uso de procesos entre estos comportamientos al hecho de que en algunos, como el tabaquismo, el cambio hace referencia a «dejar de hacer» viejos hábitos, mientras que en otros, como el ejercicio y parcialmente las dietas, el cambio hace referencia a «hacer» nuevos hábitos; en el primer caso es lógico que el hecho de dejar de pensar en los viejos hábitos sea una forma de ayudarse a cambiar, mientras que en el segundo caso el hecho de pensar en los nuevos hábitos sea una forma de ayudarse en las etapas de acción y de mantenimiento (Flórez-Alarcón, 2005).

En la línea de lo expuesto, en algunas investigaciones donde se ha utilizado el MT, han mostrado dificultades como tener que agrupar estadios (Sánchez, García, y Landabaso, 1998), o falta de consistencia interna 
(Fahrenwald y Walker, 2003; Marcus y Simkin, 1993) apoyando estos hechos la opinión de diferentes investigadores (Davidson, 2001; Hodgins, 2001; West, 2005; Wilson y Schlam, 2004) que han criticado el concepto del MT, problemas de aplicación del cuestionario, así como la forma en que se evalúan las diferentes etapas.

Usualmente se asume el movimiento de las etapas en orden, pero en realidad se trata de ciclos de etapas, siendo posible recaer en fases previas a la acción o mantenerse por mucho tiempo en la misma etapa, destacando que el tiempo de permanencia solo es determinado por el cambio comportamental del sujeto. Por ello, la motivación va a ser un elemento determinante dentro de este modelo para que se produzca el cambio (Aspano, Lobato, Leyton, Batista, y Jiménez, 2016; Ingo, Brännström, Andersson, Lunner, y Laplante-Lévesque, 2016; Jiménez, Moreno, Leyton, y Claver, 2015). Deci y Ryan (2012) y Vallerand (2015), definen la teoría de la autodeterminación como una teoría empírica de la motivación humana y la personalidad en los contextos sociales, que distingue entre motivación no autodeterminada o autodeterminada. Son numerosos los estudios que muestran que una mayor motivación autodeterminada se relaciona positivamente con un mayor compromiso y adherencia a la práctica deportiva, y por lo tanto con las etapas más activas del cambio del ejercicio, acción y mantenimiento (Aspano, et al., 2016; Buckworth, Lee, Regan, Schneider, y DiClemente, 2007; Landry y Solmon, 2004).

En este sentido, las formas de motivación más autodeterminadas se han mostrado como un factor importante para la intención de seguir siendo físicamente activo, y, esa intención va a predecir las etapas de cambio más activas (Almagro, Sáenz-López, González-Cutre, y Moreno-Murcia, 2011; Cecchini, Fernández-Losa, González, y Cecchini, 2013 ). Según Franco, Coterón, y Pérez-Tejero (2016), la intención de práctica futura está influenciada por factores motivacionales, actitudinales y comportamentales del individuo.

Por ello, el objetivo de este estudio fue traducir y validar al español el CMTCEJ, además de realizar adaptaciones y modificaciones del mismo, de manera que se pueda aplicar a toda la población, en relación a la práctica de ejercicio físico. Para buscar evidencias externas de validez, como objetivo secundario se analizaron los factores de los estadios de cambio que predicen la intención de ser físicamente activo. En este sentido, se planteó que los estadios más activos del MT (acción y mantenimiento) predecirán de manera positiva y significativa la Intención de ser Físicamente Activo (IFA).

\section{MATERIAL Y MÉTODOS}

\section{Participantes}

Tomaron parte de este estudio 812 personas de edades comprendidas entre los 14 y los 60 años $(29,5 \pm 21,7)$ de una provincia española, de los cuales 311 eran hombres y 501 mujeres. La muestra fue extraída de diferentes grupos de gimnasia de mantenimiento, así como estudiantes de secundaria y estudiantes de la carrera de Ciencias del Deporte. Los componentes de este 
grupo muestral fueron seleccionados mediante un muestreo no probabilístico intencional por conglomerado (Azorín y Sánchez-Crespo, 1986).

\section{Traducción y adaptación del instrumento}

Siguiendo a Hambleton (1996) se realizó una traducción inversa de los ítems del cuestionario URICA-E2. En primer lugar se tradujeron al castellano, y posteriormente un traductor ajeno al grupo de investigación los volvió a traducir al inglés, observando una gran similitud con el cuestionario original en habla inglesa. A continuación, los ítems fueron evaluados por cuatro expertos en la materia (Lynn, 1986), psicólogos, que consideraron que eran adecuados para evaluar el constructo para el que se creó. Una vez traducido, se administró el cuestionario a un pequeño grupo de personas con edades similares a la muestra final del estudio para verificar su correcta comprensión. No se notificaron problemas de comprensión lectora.

\section{Variables e Instrumentos de Medida}

El Cuestionario original del MTCEJ fue presentado inicialmente en un estudio con fumadores por Prochaska y DiClemente (1983), y posteriormente adaptado para el uso relacionado con la actividad física y el ejercicio por Marcus et al. (1992). Sin embargo, debido a los problemas de aplicación del cuestionario original comentado en la introducción, y con el fin de solventar este problema, el CMTCEJ se dividió en dos partes. En la primera parte, además, el estadio de precontemplación se dividió en dos factores para su mejor entendimiento por parte del sujeto. Estos fueron precontemplación no (no tiene intención de realizar ejercicio físico) y precontemplación sí (no tiene intención de realizar ejercicio físico pero lo considera importante). La primera parte hace referencia a las etapas de cambio menos activas (precontemplación no, precontemplación sí, contemplación y preparación) orientadas únicamente a las personas que no practican ejercicio físico; y la segunda parte hace referencia a las etapas de cambio más activas (acción y mantenimiento) encaminada de manera exclusiva a las personas que ya realizan ejercicio físico.

EI CMTCEJ, utiliza 22 ítems (Anexo I), para las personas que no son físicamente activas, los cuales van precedidos del encabezado "No realizo ejercicio físico regular..."; de los que se extrae 4 factores: estadio de precontemplación no (no le importan el Ejercicio Físico, compuesto por 6 ítems) (e.g. "Porque creo que no lo necesito"), estadio de precontemplación sí (reconocen que es bueno, compuesto por 5 ítems) (e.g. "Pero creo que merece la pena realizarlo"), estadio de contemplación (compuesto por 5 ítems) (e.g. "Pero me gustaría probar con alguna actividad física"), y estadio de preparación (compuesto por 6 ítems) (e.g. "Ya sé dónde voy a realizar ejercicio físico."); así como 9 ítems más (Anexo I) para las personas físicamente activas, que van precedidos del encabezado "Realizo ejercicio físico regular...", de los que se extrae 2 factores: estadio de acción (compuesto por 4 ítems) (e.g. "Recientemente he empezado a hacer ejercicio regular"), y estadio de mantenimiento (compuesto por 5 ítems) (e.g. "Llevo mucho tiempo haciendo ejercicio físico regular y pretendo seguir así."). Las respuestas fueron recogidas 
en una escala tipo Likert, cuyo rango de puntuación oscilaba entre 1 (Totalmente en desacuerdo) y 5 (Totalmente de acuerdo).

Se aplicó también la Medida de la Intencionalidad para Ser Físicamente Activo (MIFA), versión adaptada y traducida al español por Moreno et al. (2007), de Hein, Müür, y Koka (2004), denominada Intention to be Physically Active Scale (Anexo II), ya que según la bibliografía, se espera una asociación positiva con los estadios más avanzados del MT, como son los estadios de Acción y Mantenimiento. Está formada por un solo factor compuesto por 5 ítems (e.g. "Me gusta practicar deporte"). Todos los ítems se responden a través de una escala tipo Likert cuyo rango va desde el valor 0 (totalmente en desacuerdo) a 5 (totalmente de acuerdo).

\section{Procedimiento}

Para la recogida de la información, nos pusimos en contacto con el equipo directivo de los diferentes centros para pedirles su colaboración en este estudio. Se solicitó a los participantes una autorización por escrito, y a algunos de sus padres debido a su minoría de edad. La administración de las escalas definitivas se realizó en presencia del investigador principal, para explicar de forma breve los objetivos y estructura, así como la forma de rellenarlas. Durante el proceso de cumplimentación el investigador principal solventó todos aquellos problemas que pudieron surgir. El tiempo empleado en la cumplimentación del cuestionario por parte de cada participante fue de diez minutos aproximadamente.

\section{Análisis de datos}

Se llevó a cabo un análisis inicial de las propiedades psicométricas de las escalas, comprobando así su fiabilidad y validez. En primer lugar se realizó un análisis de consistencia interna a través del alfa de Cronbach. Para comprobar si la estructura de los respectivos factores, con sus correspondientes ítems, se ajustaba correctamente al contexto español se realizó el análisis factorial confirmatorio (AFC). El programa utilizado para el AFC fue el EQS 6.1 for Window- Multivariate Software, Inc.

Se consideraron una serie de índices de bondad de ajuste. Así, basándonos en las aportaciones de diferentes autores (McDonald y Marsh, 1990; Mulaik, et al., 1989; Tucker y Lewis, 1973), los índices que se consideraron para evaluar la bondad del modelo de medición fueron: $X^{2}, X^{2} / \mathrm{g} . \mathrm{l}$., RMSEA (Root Mean Square Error of Aproximation), RMSR (Root Mean Square Residual) y los índices incrementales (IFI, CFI y TLI).

Por último para determinar la validez predictiva, se realizó un análisis de regresión lineal utilizando la técnica de pasos sucesivos, empleando los factores del CMTCEJ y de la MIFA. 
El programa utilizado para el análisis de los datos obtenidos fue el programa estadístico IBM SPSS Statistics for Windows, Versión 19.0 (Armonk, NY. USA).

\section{RESULTADOS}

\section{Análisis factorial confirmatorio}

Se realizó un análisis factorial confirmatorio para evaluar el modelo de seis factores de la versión original del CMTCEJ. En el análisis, se tuvieron en cuenta una combinación de índices, tal y como aconseja Bentler (1995). Por este motivo, para determinar el ajuste de la escala se siguieron los indicadores recomendados por Byrne (2008): $X^{2}, X^{2} / g . I$., CFI (Comparative Fit Index), IFI (Incremental Fit Index), TLI (Tucker Lewis Index), RMSEA (Root Mean Square of Approximatión) y SRMR (Standardized Root Mean Square Residual). El $X^{2}$ indica el parecido de las covarianzas observadas con aquellas que se pronostican en el modelo hipotético, pero es muy sensible al tamaño de la muestra, por lo que autores como Jöreskog y Sörbom (1993) recomiendan que además se complete con el $\mathrm{x}^{2} / \mathrm{g} . \mathrm{l}$., cuyos valores por debajo de 2 indican un muy buen ajuste del modelo, mientras que valores por debajo de 5 son considerados como aceptables (Schumacker y Lomax, 2004). Los índices incrementales (CFI, IFI, TLI) comparan el modelo hipotético y el modelo nulo, no viéndose afectados por el tamaño de la muestra. Se consideran aceptables valores superiores a 0,90 (Schumacker y Lomax, 2004). Los índices de error RMSEA y SRMR deben ser inferiores a 0,08 (Browne y Cudeck, 1993; Hu y Bentler, 1999).

Se eliminaron los ítems que tenían menos pasos para conseguir un ajuste satisfactorio. Las cargas factoriales estandarizadas de los ítems con más peso, fueron estadísticamente significativas $(p<0,01)$, por lo que se puede concluir que el modelo presenta unos resultados satisfactorios (ver Tabla 1).

Tabla 1. Cargas Factoriales Estandarizadas del Cuestionario del Modelo Transteórico del Cambio de Ejercicio Físico (CMTCEJ).

\begin{tabular}{ccc}
\hline Factor & Ítems & $C F$ \\
\hline Precontemplación & 1 & $0,53^{*}$ \\
\hline
\end{tabular}




\begin{tabular}{|c|c|c|}
\hline No & 3 & $0,56^{*}$ \\
\hline & 7 & $0,49^{*}$ \\
\hline \multirow{3}{*}{$\begin{array}{c}\text { Precontemplación } \\
\text { Sí }\end{array}$} & 2 & $0,66^{*}$ \\
\hline & 8 & $0,03^{*}$ \\
\hline & 10 & $0,44^{*}$ \\
\hline \multirow{3}{*}{ Contemplación } & 5 & $0,93^{*}$ \\
\hline & 11 & $0,88^{*}$ \\
\hline & 13 & $0,36^{*}$ \\
\hline \multirow{3}{*}{ Preparación } & 4 & $0,61^{*}$ \\
\hline & 6 & $0,78^{*}$ \\
\hline & 9 & $0,69^{*}$ \\
\hline \multirow{3}{*}{ Acción } & 2 & $0,76^{*}$ \\
\hline & 5 & $0,83^{*}$ \\
\hline & 6 & $0,82^{*}$ \\
\hline \multirow{3}{*}{ Mantenimiento } & 1 & $0,87^{*}$ \\
\hline & 3 & $0,61^{*}$ \\
\hline & 4 & $0,87^{*}$ \\
\hline
\end{tabular}

Nota. CF: Carga Factorial Estandarizada; ${ }^{*} p<0,01$

Los resultados globales del modelo indicaron un ajuste óptimo: $X^{2} / \mathrm{g} . \mathrm{I}=$ $4,3, \mathrm{CFI}=0,92, \mathrm{IFI}=0,92, \mathrm{TLI}=0,90, \mathrm{RMSEA}=0,06, \mathrm{SRMR}=0,05$. Con estos resultados se puede concluir que el modelo estructural tiene un ajuste global satisfactorio.

\section{Análisis descriptivo}

Los estadísticos de media y desviación típica para cada uno de los ítems válidos del CMTCEJ y del MIFA se muestran en la tabla 2. En el CMTCEJ, las medias más altas se corresponden con el factor mantenimiento $(M=3,63$; d.t.= $1,41)$ y las medias más bajas corresponden al factor precontemplación no $(M=$ 1,75; d.t.=0,62). El MIFA, mostró una media de 3,85 (d.t.=0,88).

Tabla 2. Estadísticos Descriptivos y Fiabilidad, de los Factores del Cuestionario del Modelo Transteórico del Cambio de Ejercicio Físico (CMTCEJ) y de la Medida de la Intencionalidad para Ser Físicamente Activo (MIFA).

$$
M \quad D T \quad \text { Alfa de }
$$




\begin{tabular}{cccc}
\cline { 2 - 4 } & & & Cronbach \\
\hline Precontemplación No & 1,75 & 0,62 & 0,70 \\
\hline Precontemplación Sí & 2,21 & 0,45 & 0,71 \\
\hline Contemplación & 3,76 & 0,78 & 0,71 \\
\hline Preparación & 3,38 & 0,93 & 0,75 \\
\hline Acción & 3,23 & 1,27 & 0,71 \\
\hline Mantenimiento & 3,63 & 1,41 & 0,72 \\
\hline MIFA & 3,85 & 0,88 & 0,82 \\
\hline
\end{tabular}

\section{Análisis de consistencia interna}

Tras el análisis, los ítems quedaron agrupados de la siguiente manera: precontemplación no $(1,3,7)$, precontemplación sí $(2,8,10)$, contemplación $(5$, $11,13)$, preparación $(4,6,9)$, acción $(2,5,6)$ y mantenimiento $(1,3,4)$. La consistencia interna de cada uno de los factores resultantes del análisis factorial (alfa de Cronbach), presentó los siguientes resultados: $(0,70)$ precontemplación no, $(0,71)$ precontemplación sí, $(0,71)$ contemplación, $(0,75)$ preparación, $(0,71)$ acción, y $(0,72)$ mantenimiento. Tanto en el CMTCEJ como en la MIFA, superan el valor criterio de 0,70 establecido por Nunnally (1978) para establecer una consistencia interna aceptable (ver Tabla 1).

\section{Validez Predictiva}

En la tabla 3 se muestran los análisis de regresión lineal efectuados (utilizando la técnica de pasos sucesivos), siendo las variables predictoras las dimensiones del MT y la variable criterio la IFA. Este procedimiento ha sido utilizado para identificar aquellos factores del MT que permiten pronosticar el valor de la variable IFA, indicando la variable con mayor capacidad de predicción y el modelo más adecuando. Las variables excluidas no están presentes por falta de significancia $(p>0,05)$. Los resultados cumplen los supuestos de aceptación del modelo, como la linealidad en la relación entre variables predictoras y criterio, así como la homocedasticidad y distribución normal de los residuos, cuyo valor medio es 0 y la desviación típica prácticamente $1(0,99)$. Además, los valores de Durbin-Watson son adecuados $(1,73)$. Pardo y Ruiz (2005) consideran que cuando el estadístico se encuentra entre 1,5 y 2,5 se puede asumir que los residuos son independientes, cumpliéndose el supuesto de independencia de las variables independientes con respecto a la dependiente. Por otro lado, los estadísticos de colinealidad indican valores adecuados de índice de Tolerancia (entre 0,61 y 1,00) y de inflación de la varianza (entre 1,00 y 1,65) (Hair, Anderson, Tatham y Black, 1999).

Tabla 3. Análisis de Regresión Lineal (pasos sucesivos)

$\begin{array}{ccccccccc}\begin{array}{c}\text { Validez } \\ \text { Criterio } \\ \text { (IFA) }\end{array} & M & R & R^{2} & D-W & \begin{array}{c}\text { Variables } \\ \text { Predictoras } \\ \text { (MT) }\end{array} & B & \text { Sig. } & T \\ \end{array}$




\begin{tabular}{|c|c|c|c|c|c|c|c|c|c|}
\hline \multirow[t]{3}{*}{$\begin{array}{l}\text { Intención } \\
\text { de ser } \\
\text { Físicamen } \\
\text { te Activo }\end{array}$} & 1 & 0,46 & 0,20 & \multirow[t]{3}{*}{1,73} & Acción & 0,46 & $\begin{array}{l}37,25^{\star \star \star} \\
14,65^{\star \star \star} \\
32,17^{\star \star \star}\end{array}$ & 1,00 & 1,00 \\
\hline & 2 & 0,64 & 0,41 & & Acción & 0,10 & $2,94^{\star *}$ & 0,61 & 1,65 \\
\hline & & & & & Mantenimiento & 0,57 & $16,76^{\star * *}$ & 0,61 & 1,65 \\
\hline
\end{tabular}

Nota. IFA= Intención de ser Físicamente Activo; MT= Modelo Transteórico.

$\mathrm{M}=$ Modelo; $\mathrm{D}-\mathrm{W}=$ Durbin-Watson; $\mathrm{B}=$ Beta; $\mathrm{T}$ = Índice de Tolerancia; FIV = Factor de Inflación de la Varianza. ${ }^{*} p<0,05 ;{ }^{* *} p<0,01 ;{ }^{* * *} p<0,001$

Tal y como se puede observar en la Tabla 3, los análisis indican que el mejor modelo para explicar la intención de ser físicamente activo ha incluido a los predictores Acción y Mantenimiento. Por consiguiente, las variables que predicen significativamente y positivamente la intención de ser físicamente activo son los estadios de acción y mantenimiento, con un porcentaje de varianza explicada del $41 \%$.

\section{DISCUSIÓN}

En los últimos años se ha comprobado un crecimiento en la publicación de trabajos de adaptación y validación de cuestionarios del ámbito de la psicología del deporte en España (Cantón, Espejo y Checa, (2015); Gálvez y Morales, (2015); González, et al., (2015b); Trigueros, et al., (2016). Eso se atribuye a que tanto para la investigación como para el trabajo de campo resulta imprescindible contar con instrumentos validados adaptados al idioma y a la cultura de los usuarios.

En este estudio se ha validado la versión española del CMTCEJ siguiendo un proceso protocolarizado de traducción y adaptación y posteriormente analizando sus propiedades psicométricas en un estudio con personas practicantes de ejercicio físico.

Se ha garantizado la equivalencia cultural y lingüística de la escala con su versión original, además, los resultados presentados confirman las propiedades psicométricas y la fiabilidad de la versión en español del CMTCEJ y confirman que es una herramienta útil para determinar los estadios de cambio hacia la práctica de ejercicio físico en diferentes grupos poblacionales, desde los 14 a los 60 años.

EI CMTCEJ tiene aplicación a toda la población. Ello contrasta con otros instrumentos diseñados previamente para la evaluación de los estadios de cambio hacia la práctica de ejercicio físico, como el cuestionario de Kearney, de Graaf, Damkjaer, y Engstrom (1999), escala que fue traducida al castellano en la tesis de Montil (2004), y que se realizó para aplicarla exclusivamente a niños/as entre los 10 y los 13 años.

Un dato evidente es la falta de validación al español de cuestionarios relacionados con las etapas de cambio. González et al., (2015) emplearon la "Encuesta de Etapas de Cambio" en la planificación de profesores de educación física, basada en el MT (Buckworth, Dishman, O’Connor, y Tomporowski, 2013; 
Buckworth, et al., 2007; DiClemente, Schlundt, y Gemmell, 2015). Sin embargo, no existe ninguna evidencia de estar validada.

En la misma línea, Morínigo y Samudio (2015), utilizaron una encuesta "Etapas de Prockasca" que recogía los 5 estadios de cambio en relación a los estilos de vida, incluyendo actividad física y dieta. Realizaron la encuesta basándose en el modelo propuesto por James Prochaska en 1979.

Muchos de los cuestionarios validados han hecho referencia a conductas adictivas como consumo de alcohol, y un ejemplo es el de Autoeficacia para Abstinencia al Alcohol, construida por Flórez-Alarcón (2001) a partir de la adaptación española de la Escala General de Autoeficacia diseñada por Babler y Schwarzer (1996). Esta escala está compuesta por 20 ítems que evalúan las actitudes del sujeto respecto a sí mismo en relación con su capacidad para afrontar las tentaciones que lo invitan a consumir. Dicha adaptación se hizo bajo los conceptos del MT de Prochaska, y de las Etapas de cambio para fumadores de Velicer et al. (1995). Esta escala pertenece al cuestionario del $\mathrm{MT}$, está compuesta por tres preguntas con opción múltiple de respuesta, que permite ubicar a la persona en cualquiera de las etapas del cambio.

Como objetivo secundario, se persiguió dotar de validez predictiva al CMTCEJ por medio de un análisis de regresión por pasos sucesivos. Los datos obtenidos permitieron corroborar la hipótesis planteada ya que los estadios más activos (acción y mantenimiento) predijeron de manera positiva y significativa la IFA. El resto de los estadios (precontemplación no, precontemplación si, contemplación y preparación) no predijeron la IFA.

Son escasos los estudios que relacionan los estadios más activos del MT con la IFA. Sin embargo, según Gurrola, et al. (2013), las personas que se encuentran en la etapa de acción ya han cambiado su conducta, y empiezan a hacer la cantidad de ejercicio recomendado invirtiendo tiempo y energía en este cambio. Es decir, han hecho cambios en pro de un ambiente que los motiva a estar físicamente activos. Quienes se encuentran en la etapa de mantenimiento trabajan para no recaer y para consolidar los beneficios obtenidos. Una vez que han estado ejercitándose por seis meses en la etapa de acción, pasan a la de mantenimiento.

En este sentido, en el estadio de acción el individuo ha hecho modificaciones manifiestas y perceptibles en su forma de vida al menos por seis meses (Prochaska, Redding, y Evers, 1997). Es decir, practican ejercicio físico, y ha habido un cambio en el comportamiento que ha durado menos de seis meses. En el estadio de mantenimiento con respecto a la práctica de ejercicio físico, los individuos trabajan para sostenerse en el compromiso, prevenir la recaída y consolidar los beneficios asegurados. Quienes se encuentran en esta etapa se distinguen de los que refieren niveles más altos de autoeficacia y, sobre todo, son considerados con menor probabilidad de recaída (Prochaska y DiClemente, 1984).

El MT permite clasificar a la población en función de su grado de 
adherencia a la práctica de ejercicio físico, teniendo en cuenta no sólo la intención de práctica (Práxedes, et al., 2016), por lo tanto, resulta lógica la regresión positiva y significativa entre los estadios más activos del MT y la IFA, pues el hecho de haber alcanzado estos estadios de comportamiento con respecto a la práctica de ejercicio físico manifiesta la IFA.

Son varias las limitaciones del estudio que deberían tomarse en consideración para futuras investigaciones; en primer lugar, el hecho de haber pretendido representar la máxima diversidad de población hace que, de algún modo, el grado de práctica de ejercicio físico y el tipo también sea variable entre participantes, ya que la muestra incluye población desde los 14 a los 60 años. Otra limitación es la falta de evidencias para la validez convergente y divergente de la escala.

Por otro lado, el vocabulario empleado en los enunciados del CMTCEJ es correcto, comprensible y su extensión adecuada. Sin embargo, se recomienda, asegurar su comprensión con los participantes antes de iniciar la administración de los ítems específicos.

En futuras investigaciones sería interesante completar los análisis en relación a otras propiedades del cuestionario tales como la fiabilidad test-retest, - la sensibilidad a programas de intervención para aumentar la práctica de ejercicio físico. Para conseguir maximizar la adherencia futura de las personas hacia la práctica de ejercicio físico, es requisito imprescindible conocer el estadio en el que se encuentran así como las variables que podrían estar interviniendo a la hora de que las personas decidan adoptar dicho comportamiento, con el claro propósito de establecer posibles pautas de intervención que ayuden a los profesionales del sector.

\section{CONCLUSIONES}

En definitiva, este estudio ha permitido proporcionar un cuestionario válido y fiable para evaluar el estadio en el que se encuentran las personas con respecto a la práctica de ejercicio físico. No obstante, las propiedades psicométricas del instrumento deberían ser testadas en otros contextos deportivos quizás más específicos como el del deporte escolar, la práctica deportiva no federada o el ejercicio físico en centros deportivos, puesto que el proceso de validación de un instrumento debe ser continuado.

\section{REFERENCIAS BIBLIOGRÁFICAS}

Almagro, B. J., Sáenz-López, P., González-Cutre, D., \& Moreno-Murcia, J. A. (2011). Clima motivacional percibido, necesidades psicológicas y motivación intrínseca como predictores del comportamiento deportivo en adolescentes. International $\begin{array}{llll}\text { Journal of } & \text { Sport }\end{array}$ https://doi.org/10.5232/ricyde2011.02501

Armitage, Ch., Sheeran, P., Conner, M., \& Arden, M. A. (2004). Stages of change or changes of stage? Predicting transitions in transtheoretical model stages in relation to healthy food choice. Journal of Consulting and Clinical Psychology, 72(3), 491- 
499.

https://doi.org/10.1037/0022-006X.72.3.491

Aspano, M. I., Lobato, S., Leyton, M., Batista, M., \& Jiménez, R. (2016). Predicción de la motivación en los estadios de cambio de ejercicio más activos. Retos: Nuevas Tendencias en Educación Física, Deporte y Recreación, 30(1), 87-91.

Azorín, F., \& Sánchez-Crespo, J. L. (1986). Métodos y aplicaciones del muestreo. Madrid: Alianza Universidad.

Babler, L., \& Schwarzer, R. (1996). Evaluación de la autoeficacia: Adaptación española de la escala de autoeficacia general. Ansiedad y Estrés, 1(1), 1-8.

Bentler, P. (1995). EQS structural equations program manual. Los Ángeles: BMDP Statistical Software.

Betancurth, D. P., Álvarez, C. V., \& Vargas, L. J. (2015). Validación de contenido y adaptación del cuestionario Fantastico por técnica Delphi. Salud Uninorte, 31(2).

Browne, M. W., \& Cudeck, R. (1993). Alternative ways of assessing model fit. En K. A. Bollen y J. S. Long (Eds.), Testing structural equation models (pp. 136-162). Newbury Park, California: Sage.

Buckworth, J., Dishman, R. K., O'Connor, P. J., \& Tomporowski, P. D. (2013). Exercise Psychology. USA: Human Kinetics.

Buckworth, J., Lee, R., Regan, G., Scheider, L. K., \& DiClemente, C. (2007). Decomposing intrinsic and extrinsic motivation for exercise: Application to stages of motivational readiness. Psychology of Sport Exercise, 8(4), 441-461. https://doi.org/10.1016/i.psychsport.2006.06.007

Byrne, B. M. (2008). Testing for multigroup equivalence of a measuring instrument: A walk through the process. Psichothema, 20, 872-882.

Cantón, E., Espejo, B., \& Checa, I. (2015). Evidencias de validez convergente y testcriterio en la aplicación del Instrumento de Evaluación de Emociones en la Competición Deportiva. Revista de psicología del deporte, 24(2), 0311-316.

Casado-Pérez, C., Hernández-Barrera, V., Jiménez-García, R., Fernández-de-LasPeñas, C., Carrasco-Garrido, P., \& Palacios-Ceña, D. (2015). [Physical activity in adult working population: Results from the European National Health Survey for Spain (2009).]. Atencion primaria/Sociedad Espanola de Medicina de Familia y Comunitaria.

Catalán, A., Verdú, J., Grau, M., Iglesias, M., Val, J., Consola, A. \& Comín, E. (2014). Prevalencia y control de factores de riesgo cardiovascular en la población general: ¿qué nos dice la historia clínica electrónica?. Atención Primaria, 46(1), 15-24. https://doi.org/10.1016/i.aprim.2013.06.004

Cecchini, J. A., Fernández-Losa, J. L., González, G., \& Cecchini, C. (2013). Aplicaciones del modelo de autodeterminación en la educación física de primaria. Revista Latinoamericana de Psicología, 45(1), 97-109.

Cheung, C., Wyman, J., Gross, C., Peters, J., Findorff, M., \& Stock, H. (2006). Exercise Behavior in Older Adults: A test of the Transtheretical Model. Journal of Ageing and Physical Activity, 15(1), 103-118. https://doi.org/10.1123/japa.15.1.103

Cofán, M. (2014). Mecanismos básicos: Absorción y excreción de colesterol y otros esteroles. Clínica e Investigación en Arteriosclerosis, 26(1), 41-47. https://doi.org/10.1016/j.arteri.2013.10.008

Davidson, R. (2001). The cycle of change. Drug and Alcohol Findings, 5(1), 19-24.

Deci, E. L., \& Ryan, R. M. (2012). Self-determination theory. En A. W. Kruglanski, P. A. M. Van Lange y E. T. Higgins (Eds.), Handbook of theories social psychology (416437). London: https://doi.org/10.4135/9781446249215.n21

Esparza, Ó. A., Carrillo, I. C., Quiñones, J., Del Castillo, A., Guzmán, R. M. E., Ochoa, S. G., Gurrola, G. M., Balcázar, P., Villarreal, M. E., \& Sánchez, J. C. (2016). 
Cuestionario de ejercicio basado en el Modelo Transteórico de la conducta en una muestra mexicana. Enseñanza e Investigación en Psicología, 18(1), 121-141.

Davidson, R. (2001). The cycle of change. Drug and Alcohol Findings, 5(1), 19-24.

DiClemente, C. C. (2005). A premature obituary for the trantheorical model: a response to West (2005). Addiction, 100(8), 1046-1048. https://doi.org/10.1111/i.1360-0443.2005.01138.x

DiClemente, C. C., \& Prochaska, J. O. (1982). Self-change and therapy change of smoking behavior: a comparison of processes of change in cessation and maintenance. Addictive Behaviors, 7(1), 133-144. https://doi.org/10.1016/0306-4603(82)90038-7

DiClemente, C. C., Prochaska, J. O., Fairhurst, S. K., Velicer, W. F., Velasquez, M. M., \& Rossi, J. S. (1991). The process of smoking cessation: An analysis of precontemplation, contemplation, and preparation stages of change. Journal of Consulting and Clinical Psychology, 59(2), 295-304. https://doi.org/10.1037/0022-006X.59.2.295

DiClemente, C. C., Schlundt, D., \& Gemmell, L. (2015). Readiness and stages of change in addiction treatment. The American Journal on Addictions, 13(2), 103-119. https://doi.org/10.1080/10550490490435777

Fahrenwald, N. L., \& Walker, S. N. (2003). Application of the Transtheoretical Model of Behavior Change to the Physical Activity Behavior of WIC Mothers, Public Health Nursing, 20(4),

307-317. https://doi.org/10.1046/.1525-1446.2003.20408.x

Flórez-Alarcón, L. (2001). Diseño e implementación de un programa, elaborado conforme al modelo transteórico para la modificación del consumo abusivo de alcohol en jóvenes estudiantes de secundaria. Conferencia por Invitación. Memorias del I Congreso Latinoamericano de Psicología de la Salud, Veracruz (México), 25 de mayo de 2001.

Flórez-Alarcón, L. (2005). Evaluación de los procesos de cambio propuestos por el modelo transteórico, en estudiantes de secundaria y universitarios consumidores de alcohol. Acta Colombiana de Psicología, 13(1), 47-78.

Franco, E., Coterón, J., \& Pérez-Tejero, J. (2016). Intención de ser físicamente activos entre estudiantes de EF: diferencias según la obligatoriedad de la enseñanza. Revista Española de Educación Física y Deportes, 414(1), 39-51.

Galve, E., Alegría, E., Cordero, A., Fácila, L., Fernández, J., \& Lluís-Ganella, C. (2014). Temas de actualidad en cardiología: riesgo vascular y rehabilitación cardiaca. Revista Española de Cardiología, 67(3), 203-210. https://doi.org/10.1016/j.recesp.2013.09.021

Gálvez, P., \& Morales, V. (2015). Desarrollo y validación del cuestionario para la evaluación de la calidad percibida en servicios deportivos.(Development and validation of a questionnaire to assess perceived quality in sports services). CCD. $\begin{array}{llll}\text { Cultura, } \quad \text { Ciencia } & \text { 5 }\end{array}$ https://doi.org/10.12800/ccd.v10i28.515

González, M. Á., Castro, D., Luis, J., Pérez García, J. A., Rodríguez, T., Leandro, J., \& Aguirre Gurrola, H. B. (2015a). Aplicación del modelo trans-teórico en la planificación docente de educación física de Nuevo León. Revista de Ciencias del Ejercicio FOD, 10(10), 1-15.

González, J. P., Sánchez-Oliva, D., González-Ponce, I., Alonso, D. A., Carretero, C. M., \& Calvo, T. G. (2015b). Adaptación y validación de un cuestionario para valorar la motivación en el contexto deportivo. Cuadernos de Psicología del Deporte, 15(3), 17-26. https://doi.org/10.4321/S1578-84232015000300002

Guiraos, J., Cabrero, J., Moreno, J. P., \& Muñoz, C. L. (2009). Revisión estructurada de los cuestionarios y escalas que miden la actividad física en los adultos mayores y 
ancianos. Gaceta Sanitaria, 23(4), 334.e1-334.e17. https://doi.org/10.1016/i.gaceta.2009.03.002

Gurrola, G. M., Balcázar, P., del Castillo, A., Villarreal, M. E., Sánchez, J. C., Esparza, O. A., Carrillo, I. C., Quiñones, J., Ochoa, S. G., \& Guzmán, R. M. E. (2013). Cuestionario de ejercicio basado en el modelo transteórico de la conducta en una muestra mexicana. Enseñanza e Investigación en Psicología, 18(1), 124-141.

Gustavo, A., \& Cabrera, A. (2000). El modelo transteórico de comportamiento en salud. Revista Facultad Nacional de Salud Pública, 18(2), 129-138.

Guzmán, A. M., Ricardo, Y. G., Retamozo, L. M., \& Soto, J. M. (2015). Etapas de cambio y actividad física en un grupo de adultos de una localidad de barranquilla. Salud en Movimiento, 3(1).

Hair, J. F., Anderson, R. E., Tatham, R. L. \& Black, W. (1999). Análisis multivariante (5a ed.). Madrid: Prentice Hall.

Hambleton, R. K., (1996). Adaptación de tests para su uso en diferentes idiomas y culturas: fuentes de error, posibles soluciones y directrices prácticas. En J. Muñiz (Ed.), Psicometría (pp. 207-238). Madrid: Universitas.

Hein, V., Müür, M., \& Koka, A. (2004). Intention to be physically active after school graduation and its relationship to three tupes of intrinsic motivation. European $\begin{array}{lll}\text { Phisical Education } \quad \text { Review, } & \text { 5-19. }\end{array}$ https://doi.org/10.1177/1356336X04040618

Herzog, Th. A., Abrams, D. B., Emmons, K. M., Linnan, L. A., \& Shadel, W. G. (1999). Do processes of change predict smoking stage movements? A prospective analysis of the Transtheoretical Model. Health Psychology, 18(4), 369-375. https://doi.org/10.1037/0278-6133.18.4.369

Hodgins, D. (2001). Processes of changing gambling behavior. Addictive Behaviors, 26(1), 121-128. https://doi.org/10.1016/S0306-4603(00)00078-2

Hu, L., \& Bentler, P. M. (1999). Cutoff criteria for fit indexes in covariance structure analysis: Conventional criteria versus new alternatives. Structural Equation Modeling,
https://doi.org/10.1080/10705519909540118

Ingo, E., Brännström, K. J., Andersson, G., Lunner, T., \& Laplante-Lévesque, A. (2016). Measuring motivation using the transtheoretical (stages of change) model: $A$ follow-up study of people who failed an online hearing screening. International $\begin{array}{llll}\text { Journal of } & \text { 5udiology, 52 } & \text { 52 }\end{array}$ https://doi.org/10.1080/14992027.2016.1182650

Jiménez, R., Moreno, B., Leyton, M., \& Claver, F. (2015). Motivación y estadios de cambio para el ejercicio físico en adolescentes. Revista Latinoamericana de Psicología, https://doi.org/10.1016/i.rlp.2014.11.001

Jöreskog, K. G., \& Sörbom, D. (1993). LISREL 8: Structural equation modeling with the SIMPLIS command language. Chicago: Scientific Software.

Kearney, J. M., de Graaf, C., Damkjaer, S., \& Engstrom, L. M. (1999). Stages of change towards physical activity in a nationally representative sample in the European Union. Public Health Nutrition, 2(1a), 115-124. https://doi.org/10.1017/S1368980099000166

Landry, J. B., \& Solmon, M. A. (2004). African American women's selfdetermination across the stages of change for exercise. Journal of Sport and Exercise Psychology, 26(3), 457-469. https://doi.org/10.1123/jsep.26.3.457

Lynn, M., (1986). Determination and quantification of content validity. Nursing Reasearch, $35(1)$, 382-385. https://doi.org/10.1097/00006199-198611000-00017

Marcus, B. H., Selby, V. C., Niaura, R. S., \& Rossi, J. S. (1992). Self-efficacy and the stages of exercise behaviour change. Research Quarterly in Exercise and Sport, 
63(1),

60-66.

https://doi.org/10.1080/02701367.1992.10607557

Marcus, B. H., \& Simkin, L. R. (1993). The stages of exercise behavior. Journal of Sports Medicine and Physical Fitness, 33(1), 83-88.

McDonald, R. P., \& Marsh, H. W. (1990). Choosing a multivariate model: non centrality and goodness of fit. Psychological Bulletin, 107, 247-255. https://doi.org/10.1037/0033-2909.107.2.247

Montil, M. (2004). Determinantes de la conducta de actividad Física en población infantil. Tesis Doctoral sin publicar. Universidad Politécnica de Madrid, Madrid, España.

Moreno, J., Cruz, H., \& Fonseca, A. (2014). Evaluación de razones de prevalencia para sedentarismo y factores de riesgo en un grupo de estudiantes universitarios. Chía, Colombia. Enfermería Global: Revista Electrónica Semestral de Enfermería, 13(2), 114-122.

https://doi.org/10.6018/eglobal.13.2.172951

Moreno, J. A., Moreno , R., \& Cervelló, E. (2007). El autoconcepto físico como predictor de la intención de ser físicamente activo. Psicología y Salud, 17(2), 261-267.

Moreno, J. A., Pardo, M., Jorge, P., \& Huéscar, E. (2016). Motivos de práctica físicodeportiva en mujeres. Revista de Psicología del Deporte, 25(1), 35-41.

Moreno, J. M., Silva, F. B., Pardo, P. M., Rodríguez, A. S., \& Hernández, E. H. (2016). Motivation, frequency and activity type in physical exercise practitioners. Revista Internacional de Medicina y Ciencias de la Actividad Física y del Deporte, 12(48), 649-662.

Morínigo, M., \& Samudio, M. (2015). Stages of the behavioral model of life styles in adults proposed by food guides of Paraguay. Memorias del Instituto de Investigaciones en Ciencias de la Salud, 13(1), 41-48. https://doi.org/10.18004/Mem.iics/1812-9528/2015.013(01)41-048

Mulaik, S. A., James, L. R., Van Astine, J., Bennett, N., Lind, S., \& Stilwell, C. D. (1989). Evaluation of goodness-of-fit indices for structural equation models. Psychological Bulletin, $105(1)$, https://doi.org/10.1037/0033-2909.105.3.430

Nunnally, J. C. (1978). Psychometric theory. New York: McGraw-Hill.

Pardo, A., \& Ruiz, M. A. (2005). Análisis de datos con SPSS 13 Base. Madrid: McGraw Hill.

Perz, C. A., DiClemente, C. C., \& Carbonari, J. P. (1996). Doing the right thing at the Right time? The interaction of stages and processes of change in successful smoking cessation. Health Psychology, 15(6), 452-468. https://doi.org/10.1037/0278-6133.15.6.462

Práxedes, A., Sevil, J., Moreno, A., del Villar, F., \& García-González, L. (2016). Niveles de actividad física en estudiantes universitarios: diferencias en función del género, la edad y los estados de cambio. Revista Iberoamericana de Psicología del Ejercicio y el Deporte, 11(1), 123-132.

Prochaska J. (1979). Systems of psychoterapy: a transtheoretical analisys. Homewood IL: Dorsey.

Prochaska, J., \& DiClemente, C. (1982). Transactional therapy: toward a more integrative model of change. En: Psichoterapy: theory, research and practice, 19(1), 276-288. https://doi.org/10.1037/h0088437

Prochaska, J. O., \& DiClemente, C. (1983). Stages and processes of self-change of smoking: toward an integrative model of change. Journal of Consulting and Clinical Psychology, $51(3)$, 390-395. https://doi.org/10.1037/0022-006X.51.3.390

Prochaska, J., \& DiClemente, C. (1984). Transtheoretical approach. New York: Dow Jones. 
Prochaska, J. O., DiClemente, C. C., Velicer, W. F., Ginpil, S., \& Norcross, J. C. (1985). Predicting change in smoking status for self-changers. Addictive Behaviors, 10(4), 395-406. https://doi.org/10.1016/0306-4603(85)90036-X

Prochaska, J. O., Norcross, J. C., Fowler, J. L., Follnick, M. J., \& Abrams, D. B. (1992). Attendance and outcome in a work site weight control program: Processes and stages of change as process and predictor variables. Addictive Behaviors, 17(1), 35-45. https://doi.org/10.1016/0306-4603(92)90051-V

Prochaska, J. O., Redding, C. A., \& Evers, K. (1997). The Transtheoretical Model and Stages of Change. En K. Glantz, F. M. Lewis y B. K. Rimer (Eds.): Health behavior and health education: Theory, research, and practice (pp.60-84). San Francisco, CA: Jossey-Bass.

Prochaska, J., \& Velicer, W. (1997). The transtheoretical model of health behavior change. American Journal of Health Promotion, 12(1), 38-48. https://doi.org/10.4278/0890-1171-12.1.38

Prochasca, J., Velicer, W., Rossi, J., Goldstein, M. G., Marcus, B. H., Rakowski, W., Fiori, C., Harlow, L. L., Redding, C. A., Rosenbloom, D., \& Rossi, S. R. (1994). Stages of change and decisional balance for 12 problem behaviors. Health Psychology, https://doi.org/10.1037/0278-6133.13.1.39

39-46.

Puigarnau, S., Foguet, O. C., Balcells, M. C., Ambrós, Q. P., \& Anguera, M. T. (2016). El apoyo a la autonomía en practicantes de centros deportivos y de fitness para aumentar su motivación. RICYDE. Revista Internacional de Ciencias del Deporte, 12(43), https://doi.org/10.5232/ricyde2016.04303

Rosen, C. S. (2000). Is the sequencing of change processes by stage consistent across health problems? A meta-analysis. Health Psychology, 19(6), 593-604. https://doi.org/10.1037/0278-6133.19.6.593

Royo-Bordonada, M., Lobos, M., Brotons, C., Villar, F., Pablo, C., Armario, P., Cortés, O., Gil, A., Lizcano, A., Santiago, A., \& Sans, S. (2014). El estado de la prevención cardiovascular en España. Medicina Clínica, 142(1), 7-14. https://doi.org/10.1016/j.medcli.2012.09.046

Sánchez, A., García, F., \& Landabaso, V. (1998). Participación en actividad física de una muestra universitaria a partir del modelo de las etapas de cambio en el ejercicio físico: un estudio piloto. Revista de Psicología del Deporte, 7(2), 233-245.

Schumacker, R., \& Lomax, R., (2004). A beginner's guide to structural equation modeling (2a ed.). Mahwah, $\mathrm{NJ}$ : Lawrence Erlbaum Associates. https://doi.org/10.4324/9781410610904

Segan, C. A., Borland, R., \& Greenwood, K. M. (2004). What is the right thing at the Right time? Interactions between stages and processes of change among smokers who make a quiet attempt. Health Psychology, 23(1), 86-93. https://doi.org/10.1037/0278-6133.23.1.86

Sotos-Prieto, M., Santos-Beneit, G., Bodega, P., Pocock, S., Mattei, J., \& Peñalvo, J. L. (2015). Validación de un cuestionario para medir los hábitos de estilo de vida mediterráneos para su aplicación en investigación: índice de estilo de vida mediterráneo (medlife). Nutrición Hospitalaria, 32(3), 1153-1163.

Trigueros, R., Aguilar-Parra, J. M., Fernández-Campoy, J. M., Manzano-León, A., \& Alcaraz-lbáñez, M. (2016, February). Validación de la ccbs en actividad física en personas mayores de 55 años de edad. In IV Congreso internacional de deporte inclusivo. Universidad de Almería 25 al 29 de mayo 2015 (Vol. 28). Universidad Almería. 
Tucker, L. R., \& Lewis, C. (1973). The reliability coefficient for maximum likelihood factor analysis.

Psychometrika,

$38(1)$,

$1-10$. https://doi.org/10.1007/BF02291170

Vallerand, R. J. (2015). The psychology of passion: A dualistic model. Oxford University Press, USA. https://doi.org/10.1093/acprof:oso/9780199777600.001.0001

Velicer, W. F., Fava, J. L., Prochaska, J. O., Abrams, D. B., Emmons, K. M., \& Pierce, J. (1995). Distribution of smokers by stage in three representative samples. Preventive Medicine, 24(1), 401-411. https://doi.org/10.1006/pmed.1995.1065

West, R. (2005). Time for a change: putting de transtheoretical (stage of change) model to rest. Addiction, 100(8), 1036-1039. https://doi.org/10.1111/j.1360-0443.2005.01139.x

Wilson, G., \& Schlam, T. (2004). The transtheoretical model and motivational interviewing in the treatment of eating and weight disorders. Clinical Psychology Review, 24(3), https://doi.org/10.1016/j.cpr.2004.03.003

361-378.

Referencias totales / Total references: 85 (100\%)

Referencias propias de la revista/Journal's own references: $0(0 \%)$

Rev.int.med.cienc.act.fís.deporte - vol. 19 - número 74 - ISSN: 1577-0354 


\section{CUESTIONARIO DEL MODELO TRANSTEÓRICO DEL CAMBIO DE EJERCICIO FÍSICO (CMTCEJ)}

NO REALIZO EJERCICIO FÍSICO REGULAR

\begin{tabular}{|l|l|lllll|}
\hline 1 & Porque creo que no lo necesito. & 1 & 2 & 3 & 4 & 5 \\
\hline 2 & Porque no tengo acceso a un lugar adecuado. & 1 & 2 & 3 & 4 & 5 \\
\hline 3 & Y estoy satisfecho de ser una persona sedentaria. & 1 & 2 & 3 & 4 & 5 \\
\hline 4 & $\begin{array}{l}\text { Pero estoy pensando que debería intentar comenzar un programa } \\
\text { de ejercicio físico regular en los próximos 6 meses. }\end{array}$ & 1 & 2 & 3 & 4 & 5 \\
\hline 5 & Pero me gustaría probar con alguna actividad física. & 1 & 2 & 3 & 4 & 5 \\
\hline 6 & $\begin{array}{l}\text { Pero ya he establecido un día y una hora para empezar a hacer } \\
\text { ejercicio físico regular en las próximas semanas. }\end{array}$ & 1 & 2 & 3 & 4 & 5 \\
\hline 7 & Y no tengo pensado hacerlo. & 1 & 2 & 3 & 4 & 5 \\
\hline 8 & Pero creo que merece la pena realizarlo. & 1 & 2 & 3 & 4 & 5 \\
\hline 9 & $\begin{array}{l}\text { Pero ya sé dónde voy a realizar ejercicio físico (gimnasio, parque, } \\
\text { pista polideportiva,...). }\end{array}$ & 1 & 2 & 3 & 4 & 5 \\
\hline 1 & Porque no puedo contar con mi entorno (familia, amigos,...). & 1 & 2 & 3 & 4 & 5 \\
\hline 1 & Pero quería comenzar a tener una vida más activa. & 1 & 2 & 3 & 4 & 5 \\
\hline 1 & Porque no tengo energía (no me apetece). & 1 & 2 & 3 & 4 & 5 \\
\hline 1 & Pero me he planteado si sería capaz de hacerlo. & 1 & 2 & 3 & 4 & 5 \\
\hline 1 & Pero he estado pensando en que quizá quiera empezar. & 1 & 2 & 3 & 4 & 5 \\
\hline 1 & Pero lo considero importante. & 1 & 2 & 3 & 4 & 5 \\
\hline 1 & Pero he estado pensando en la posibilidad de comenzar a hacerlo. \\
6 & 1 & 2 & 3 & 4 & 5 \\
\hline 1 & Y no me preocupa. & 1 & 2 & 3 & 4 & 5 \\
\hline 1 & $\begin{array}{l}\text { Pero ya me estoy preparando para empezar a hacer ejercicio en } \\
8\end{array}$ & 1 & 2 & 3 & 4 & 5 \\
\hline 1 & $\begin{array}{l}\text { Pero ya he quedado con un amigo para empezar a hacer ejercicio } \\
\text { dentro de las próximas semanas. }\end{array}$ & 1 & 2 & 3 & 4 & 5 \\
\hline 2 & $\begin{array}{l}\text { Pero ya he estado llamando a amigos para encontrar a alguien con } \\
0\end{array}$ & 1 & 2 & 3 & 4 & 5 \\
\hline 2 & quien empezar a hacer ejercicio en las próximas semanas. & 1 & 2 & 3 & 4 & 5 \\
\hline 2 & Porque no lo considero importante. & 2 & 3 & 4 & 5 \\
\hline 2 & Porque no tengo tiempo. & & & & \\
\hline & & 1 & 1 & 5
\end{tabular}


REALIZO EJERCICIO FÍSICO REGULAR...

\begin{tabular}{|l|l|lllll|}
\hline 1 & $\begin{array}{l}\text { He tenido éxito en hacer ejercicio regularmente y tengo pensado } \\
\text { continuar. }\end{array}$ & 1 & 2 & 3 & 4 & 5 \\
\hline 2 & Recientemente he empezado a hacer ejercicio regular. & 1 & 2 & 3 & 4 & 5 \\
\hline 3 & $\begin{array}{l}\text { Llevo mucho tiempo haciendo ejercicio físico regular y pretendo } \\
\text { seguir así. }\end{array}$ & $\begin{array}{l}1 \\
2\end{array}$ & 3 & 4 & 5 \\
\hline 4 & $\begin{array}{l}\text { He conseguido seguir haciendo ejercicio físico en los últimos seis } \\
\text { meses. }\end{array}$ & 3 & 4 & 5 \\
\hline 5 & He empezado a hacer ejercicio regular y tengo pensado continuar. & 1 & 2 & 3 & 4 & 5 \\
\hline 6 & $\begin{array}{l}\text { He empezado a hacer ejercicio con regularidad en los últimos seis } \\
\text { meses. }\end{array}$ & 1 & 2 & 3 & 4 & 5 \\
\hline 7 & Por fin hago ejercicio con regularidad. & 1 & 2 & 3 & 4 & 5 \\
\hline 8 & Llevo tiempo haciendo ejercicio y tengo pensado continuar. & 1 & 2 & 3 & 4 & 5 \\
\hline 9 & He completado más de seis meses haciendo ejercicio físico regular. & 1 & 2 & 3 & 4 & 5 \\
\hline
\end{tabular}

Estadio de precontemplación (no le importa el ejercicio físico): 1-3-7-1217-21

Estadio de precontemplación (reconoce que el ejercicio físico es bueno): 2-8-10-15-22

Estadio de contemplación: 5-11-13-14-16

Estadio de preparación: 4-6-9-18-19-20

Estadio de acción: 2-5-6-7

Estadio de mantenimiento: 1-3-4-8-9

Rev.int.med.cienc.act.fís.deporte - vol. 19 - número 74 - ISSN: 1577-0354

ANEXO II 
MEDIDA DE LA INTENCIONALIDAD PARA SER FÍSICAMENTE ACTIVO (MIFA)

\begin{tabular}{|l|l|rrrrr|}
\hline 1 & Me interesa el desarrollo de mi forma física. & 1 & 2 & 3 & 4 & 5 \\
\hline 2 & Me gusta practicar deporte. & 1 & 2 & 3 & 4 & 5 \\
\hline 3 & Quisiera formar parte de un grupo para realizar deporte. & 1 & 2 & 3 & 4 & 5 \\
\hline 4 & Me gustaría mantenerme físicamente activo/a. & 1 & 2 & 3 & 4 & 5 \\
\hline 5 & Habitualmente practico actividad físico-deportiva en mi tiempo libre. & 1 & 2 & 3 & 4 & 5 \\
\hline
\end{tabular}

Rev.int.med.cienc.act.fís.deporte - vol. 19 - número 74 - ISSN: 1577-0354 